\title{
Positioning Janus Nanoparticles in Block Copolymer Scaffolds
}

\author{
Jaeup U. Kim and Mark W. Matsen \\ Department of Mathematics, University of Reading, Whiteknights, Reading RG6 6AX, United Kingdom
}

(Received 3 November 2008; published 20 February 2009)

\begin{abstract}
The periodic domains formed by block copolymer melts have been heralded as potential scaffolds for arranging nanoparticles in 3D space, provided we can control the positioning of the particles. Recent experiments have located particles at the domain interfaces by grafting mixed brushes to their surfaces. Here the underlying mechanism, which involves the transformation into Janus particles, is investigated with self-consistent field theory using a new multi-coordinate-system algorithm.
\end{abstract}

DOI: 10.1103/PhysRevLett.102.078303

Block copolymers are long-chain molecules composed of two or more chemically distinct sections (or blocks); the natural tendency for the unlike blocks to segregate causes the molecules to self-assemble into nanosized domains with periodic order. There is now an intense effort to use the resulting block-copolymer morphologies as scaffolds to position nanoparticles into ordered arrays for such applications as photonic band-gap materials, solar cells, fuel cells, optical filters, nanowires, high-efficient catalysts, and high-density magnetic storage media [1-6]. The positioning of particles is affected by their size and shape [6-9], but the single most important factor is their chemical interaction with the block copolymer [2-7,10-13]. The interaction is readily adjusted by grafting a brush of short chains to the surface of each particle [2,11-13]. Chiu et al. [11] have devised the most systematic approach using mixed brushes of variable composition to tune the interaction; in their study, the position of gold nanoparticles in a polystyrene-poly(2-vinylpyridine) (PS-P2VP) diblockcopolymer lamellar morphology was controlled with brushes of short PS and P2VP homopolymers. Not surprisingly, symmetric brush compositions positioned the particles at the domain interfaces, whereas asymmetric compositions forced the particles to the corresponding domain centers. However, the preference for the interface was much stronger than expected from simple surface tension considerations. Further studies by Kim et al. [12] showed that the interface was preferred for brush compositions of $10 \%-90 \%$ PS. A clue to what was going on emerged from the observation that the interfacial preference was significantly reduced when the mixed brush was substituted by a random-copolymer brush where each chain is a uniform mixture of PS and P2VP segments. Combining this with existing evidence [14] that the functionalized ends of the brush are able to diffuse significant distances along gold surfaces (on the time scale of their experiment), Kim et al. suggested that the unusually high interfacial affinity created by the mixed brush resulted from the grafted ends of its PS and P2VP chains segregating to opposite hemispheres forming a Janus nanoparticle as illustrated in Fig. 1. In this Letter, we develop a theoretical method
PACS numbers: 82.35.Np, 81.07.-b, 81.16.Dn, 83.80.Uv

that allows us to quantitatively investigate this proposed mechanism.

So far, theories $[15,16]$ as well as simulations $[3,13]$ have only managed to model bare particles. One of the first and most widely used theories for block-copolymernanoparticle composites combines self-consistent field theory (SCFT) for polymers with a density functional theory (DFT) for hard spheres [15], but it does not properly treat the polymer-particle interactions. This is because it allows the polymers to penetrate the space occupied by the particles. More recently, Sides et al. [13] have developed a two-step strategy that overcomes this excluded-volume problem, whereby the statistical mechanics of the polymer is performed for fixed particle positions using SCFT, producing an effective Hamiltonian for the particles which can then be treated by simulation. Unfortunately, the computational demands of the calculation are far too great to also include brushes on the particles. However, the demands can be vastly reduced by considering the idealgas limit, where particle-particle interactions can be ignored. In this limit, the particle distribution is $\rho\left(\mathbf{r}_{p}\right) \propto$ $\exp \left(-F\left(\mathbf{r}_{p}\right) / k_{B} T\right)$, where $F\left(\mathbf{r}_{p}\right)$ is the free energy of the polymers in the presence of a single impenetrable spherical particle centered at $\mathbf{r}_{p}$. This permits us to develop the first calculation capable of modeling the effect of polymer brushes on the positioning of a nanoparticle in a blockcopolymer morphology.

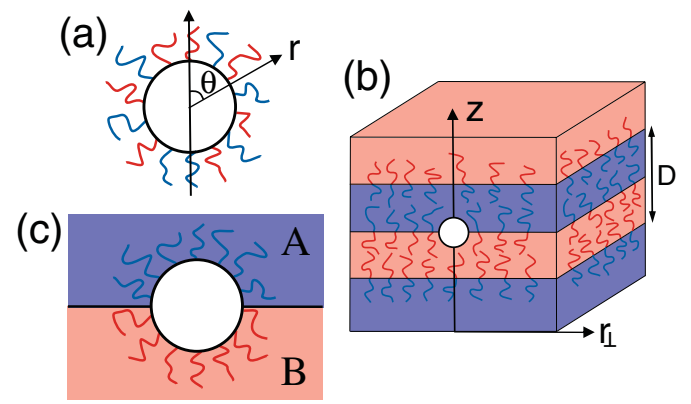

FIG. 1 (color). Schematic diagrams showing (a) a nanoparticle with a mixed brush inserted into (b) a diblock-copolymer lamellar morphology producing (c) a Janus nanoparticle. 
In our model, each diblock copolymer consists of one block of $f_{c} N A$-type segments joined to another block of $\left(1-f_{c}\right) N B$-type segments, while each brush chain has $\alpha N$ segments. For the mixed brush, the chains are made up of either all $A$ or all $B$ segments, and for the randomcopolymer brush, the chains are a uniform mixture of $A$ and $B$ segments. The $A$-segment content of either brush is specified by $0 \leq f_{b} \leq 1$. The $A$ and $B$ segments are defined so as to occupy a common volume, $\rho_{0}^{-1}$, and are assumed to have the same statistical length, $a$. Following the SCFT [17], the $A$ segments interact via a mean field,

$$
w_{A}(\mathbf{r})=\chi N \phi_{B}(\mathbf{r})+\xi(\mathbf{r}),
$$

which is determined self-consistently from the total $B$-segment concentration, $\phi_{B}(\mathbf{r})$. The Flory-Huggins $\chi$ parameter specifies the interaction strength between $A$ and $B$ segments, and $\xi(\mathbf{r})$ is a pressure field that enforces incompressibility, $\phi_{A}(\mathbf{r})+\phi_{B}(\mathbf{r})=1$. The field acting on $B$ segments is obtained by exchanging the labels, $A$ and $B$, in Eq. (1).

The SCFT requires a single-chain partition function, $Q_{i}=\int q_{i}(\mathbf{r}, s) q_{i}^{\dagger}(\mathbf{r}, s) d \mathbf{r}$, for each type of polymer, $i$. It is expressed in terms of a partial partition function, $q_{i}(\mathbf{r}, s)$, for the first $s N$ segments with the $s N$ th segment fixed at position, $\mathbf{r}$, which is evaluated by solving

$$
\frac{\partial}{\partial s} q_{i}(\mathbf{r}, s)=\left[\frac{a^{2} N}{6} \nabla^{2}-w(\mathbf{r})\right] q_{i}(\mathbf{r}, s),
$$

with the initial condition, $q_{i}(\mathbf{r}, 0)=1$. For the diblock copolymer $(i=c)$, the field switches from $w_{A}(\mathbf{r})$ for $s<$ $f_{c}$ to $w_{B}(\mathbf{r})$ for $s>f_{c}$. The $A$ brush chain $(i=b A)$ uses $w_{A}(\mathbf{r})$, the $B$ one $(i=b B)$ uses $w_{B}(\mathbf{r})$, and the randomcopolymer chain $(i=b)$ uses the mixed field, $w(\mathbf{r})=$ $f_{b} w_{A}(\mathbf{r})+\left(1-f_{b}\right) w_{B}(\mathbf{r})$. Likewise, there is an analogous function, $q_{i}^{\dagger}(\mathbf{r}, s)$, for the remaining segments of each chain, which satisfies the same diffusion equation but with the left side multiplied by -1 . For the diblock copolymer, it is solved starting again from the free-end condition, $q_{c}^{\dagger}(\mathbf{r}, 1)=1$. For the brush chains, however, it is solved using the condition, $q_{i}^{\dagger}(\mathbf{r}, \alpha)=\delta\left(\left|\mathbf{r}-\mathbf{r}_{p}\right|-\right.$ $R_{p}$ ), which constrains the grafted ends to a radius, $R_{p}$, from the particle center, $\mathbf{r}_{p}$ (i.e., to the particle surface).

Once all the partition functions have been evaluated, the $A$-segment concentration is given by

$$
\begin{aligned}
\phi_{A}(\mathbf{r})= & \frac{V_{c}}{Q_{c}} \int_{0}^{f_{c}} q_{c}(\mathbf{r}, s) q_{c}^{\dagger}(\mathbf{r}, s) d s \\
& +\frac{f_{b} V_{b}}{\alpha Q_{i}} \int_{0}^{\alpha} q_{i}(\mathbf{r}, s) q_{i}^{\dagger}(\mathbf{r}, s) d s,
\end{aligned}
$$

where $i=b A$ for the mixed brush and $i=b$ for the random-copolymer brush. $V_{c}$ and $V_{b}$ are total volumes occupied by the diblock copolymer and brush, respectively. The $B$ polymer concentration is given by an analogous expression. Once the fields have been adjusted to satisfy the self-consistent conditions [e.g., Eq. (1)], the free energy for the system with a mixed brush is

$$
\begin{aligned}
\frac{N F\left(\mathbf{r}_{p}\right)}{\rho_{0} k_{B} T}= & -\frac{f_{b} V_{b}}{\alpha} \ln Q_{b A}-\frac{\left(1-f_{b}\right) V_{b}}{\alpha} \ln Q_{b B}-V_{c} \ln Q_{c} \\
& +\int\left(\chi N \phi_{A} \phi_{B}-w_{A} \phi_{A}-w_{B} \phi_{B}\right) d \mathbf{r} .
\end{aligned}
$$

For the system with a random-copolymer brush, the free energy is the same but with the first two logarithmic terms replaced by $-\left(V_{b} / \alpha\right) \ln Q_{b}$.

To make the problem tractable, we consider a lamellar phase such that there is axial symmetry about the center of the particle. This reduces the diffusion equation to a $2 \mathrm{D}$ calculation, which can be solved efficiently with the Crank-Nicolson finite-difference scheme as described in Ref. [16]. Even still, the geometry poses some difficulties. The only way to take advantage of the symmetry for the block copolymer is to solve the diffusion equation in a cylindrical cell using cylindrical coordinates, $\left(r_{\perp}, z, \varphi\right)$; see Fig. 1(b). This allows reflecting boundary conditions to be applied, provided that the cell is chosen large enough with its side perpendicular to the lamellae and its two flat ends coinciding with lamellar midplanes [16]. However, an efficient treatment of the brush requires that we work with spherical-polar coordinates, $(r, \theta, \varphi)$; see Fig. 1(a). This facilitates a reflecting boundary at the particle surface, $r=$ $R_{p}$, to which one end of each chain is constrained. We circumvent this dilemma by implementing a multicoordinate-system (MCS) scheme, whereby the diblockcopolymer and brush concentrations are calculated in cylindrical and spherical coordinates, respectively. The only complication is with the sharing of concentrations between the two coordinate systems. To handle the fact that their grid points do not match, the concentration at a grid point in one system is estimated from that of the four nearest points in the other system, assuming short-scale linearity.

Our system parameters are set to roughly match the experiments of Kim et al. The diblock copolymer is chosen with $\chi N=100$ and $f_{c}=0.5$, which produces a lamellar structure of period, $D=2.337 a N^{1 / 2}$. The nanoparticle is chosen to have a radius of $R_{p}=0.08 a N^{1 / 2}$ and short grafted chains of $\alpha=0.04$. The amount of polymer grafted to the particle is set to $V_{b}=4 \pi R_{p}^{3}$, which results in a brush height similar to the particle radius. For these parameters, the diblock copolymer is solved in a cylindrical cell with 400 mesh points for $r_{\perp}=0$ to $a N^{1 / 2}$, 1000 points for $z=-0.75 D$ to $+0.75 D$, and 400 points for $s=0$ to 1 . The brush is solved in a spherical shell with 188 points for $r=R_{p}$ to $0.55 a N^{1 / 2}, 300$ points for $\theta=0$ to $\pi$, and 400 points for $s=0$ to $\alpha$.

We begin by examining a nanoparticle grafted with random copolymer of symmetric composition, $f_{b}=0.5$, which prefers to locate at the lamellar interface $(z=0)$. Figure 2 shows how the polymer composition, $\phi_{A}(\mathbf{r})$, responds as the particle is moved away from its preferred location. The block-copolymer morphology is composed of highly pure domains separated by narrow interfaces, 


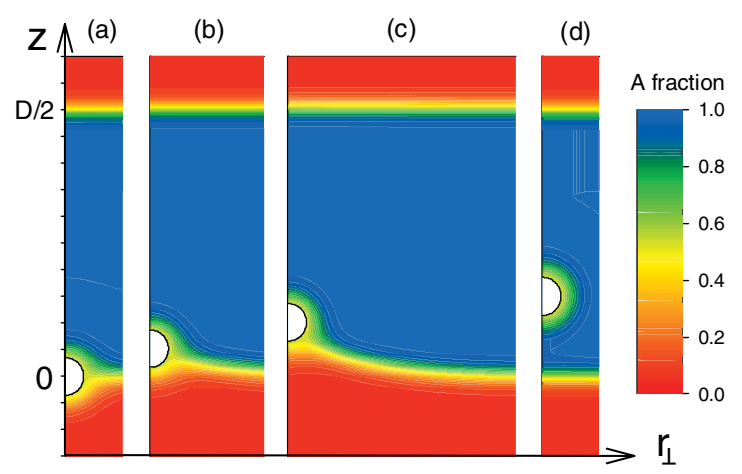

FIG. 2 (color). Total $A$-segment concentration, $\phi_{A}(\mathbf{r})$, when the nanoparticle is coated with a random-copolymer brush and positioned at (a) $z_{p} / D=0.0$, (b) 0.05 , (c) 0.10 , and (d) 0.15 .

while the brush produces a halo of intermediate concentration around the particle. As the particle is moved towards positive $z$, the interface deforms in order to follow its position, but only up to a certain point. When the interface becomes too deformed, it simply detaches from the particle.

The calculation is repeated in Fig. 3, but now for a mixed brush with equal numbers of $A$ and $B$ chains such that the composition remains $f_{b}=0.5$. The behavior is very similar to that of Fig. 2, except for the segment concentration next to the particle. While the particle is in contact with the interface, there is no longer any clear distinction between the block-copolymer and brush regions. Evidently, the $A$ and $B$ brush chains segregate so as to mimic the block copolymer morphology. Only when the interface detaches leaving the particle isolated in a chemically homogeneous background, do the brush chains mix together producing a halo of intermediate concentration around the particle.

To confirm this, Fig. 4 plots the $A$-segment distribution from just the brush [i.e., the second integral in Eq. (3)] for $z_{p}=0$. Here it is now clear that the chains of the mixed brush segregate to opposite hemispheres creating a Janus particle, whereas the random-copolymer brush remains nearly isotropic. The segregation is illustrated more quantitatively in Fig. 5(a), where the distributions of the grafted ends, $\sigma_{i}(\theta) \propto q_{i}\left(R_{p}, \theta, \alpha\right)$, of the $A$ (blue curve) and $B$ (red curve) chains are compared. Despite the segregation of unlike chains, the total distribution, $\sigma_{b}(\theta) \equiv \sigma_{b A}(\theta)+$ $\sigma_{b B}(\theta)$, is surprisingly uniform (black curve) with just slightly more chain ends at the equator $(\theta \approx \pi / 2)$ than the two poles $(\theta \approx 0$ and $\pi)$. The small variation is primarily due to the pressure field, $\xi(\mathbf{r})$, which pushes segments towards the interface in order to satisfy the incompressibility constraint. This is confirmed from the distribution of the random-copolymer ends (dashed curve), which is affected solely by the pressure field; the field acting on the random copolymer reduces to $w(\mathbf{r})=$ $\chi N / 2+\xi(\mathbf{r})$ when $f_{b}=0.5$.

Figure 5(b) shows what happens when the particle is displaced to $z_{p}=0.1 D$. As illustrated in Fig. 3(c), the interface follows the particle but its contact line slips

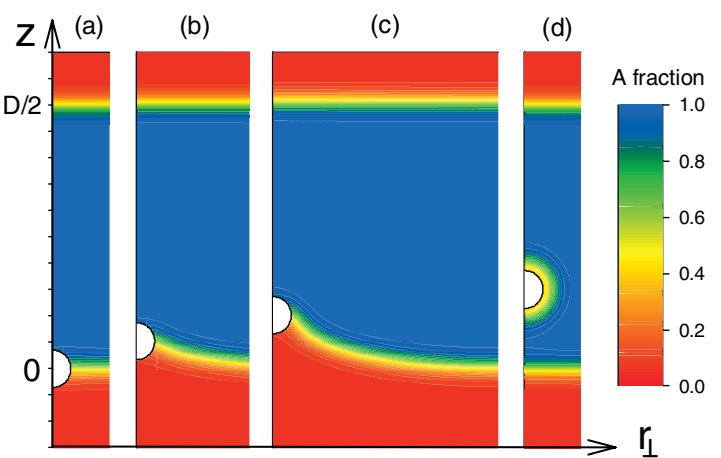

FIG. 3 (color). Analogous to Fig. 2, but with a mixed brush.

slightly away from the equator of the particle. This is reflected in the fact that the point at which $\sigma_{b A}(\theta)=$ $\sigma_{b B}(\theta)$ shifts from $\theta=0.5 \pi$ to $0.532 \pi$. Consequently, the $B$ chains are squeezed into a smaller area causing a rise in their grafting density. Interestingly, the total grafting density, $\sigma_{b}(\theta)$, develops a significant variation compared to that of Fig. 5(a). This is also true for the randomcopolymer brush (dashed curve), which as before implies that the behavior is linked to $\xi(\mathbf{r})$. The explanation is simple; as the interface in Fig. 3(c) is pulled upward, it becomes more difficult to fill the space beneath the particle with $B$ segments, which has to be compensated for by a decrease in pressure.

We now turn our attention to the particle distribution, $\rho\left(z_{p}\right) \propto \exp \left(-F\left(z_{p}\right) / k_{B} T\right)$. To evaluate $F\left(z_{p}\right) / k_{B} T$ from Eq. (4), we select an invariant polymerization index of $\bar{N} \equiv \rho_{0}^{2} a^{6} N=5000$, which roughly corresponds to the $196 \mathrm{~kg} / \mathrm{mol}$ PS-P2VP diblock copolymers in Ref. [12]. Using this value, Fig. 6(a) plots the particle distributions for mixed brushes of various compositions, $f_{b}$. Note that since the lamellar phase is symmetric (i.e., $f_{c}=0.5$ ), we only need to examine $f_{b} \geq 0.5$. The distributions predict that most particles will migrate to the interface for $0.1 \lesssim$ $f_{b} \lesssim 0.9$ and to the appropriate domain centers otherwise, which perfectly matches the experimental observations. Also consistent with the experiments, the distributions are more localized for particles at the interface than in the domains. Interestingly, small deviations from $f_{b}=0.5$ cause the particles to shift off-center from the interface, while still remaining strongly attached. In Fig. 6(b), the calculations are repeated, but this time for nanoparticles grafted by random copolymers. As expected, the range

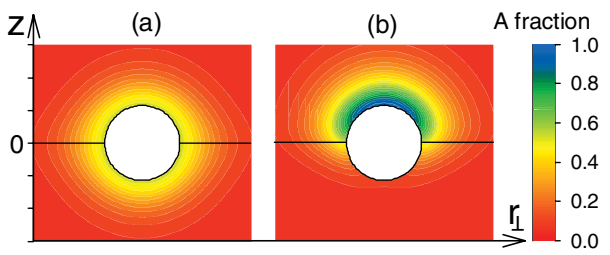

FIG. 4 (color). A-segment concentration from just the (a) random-copolymer and (b) mixed brushes, corresponding to Figs. 2(a) and 3(a), respectively. 

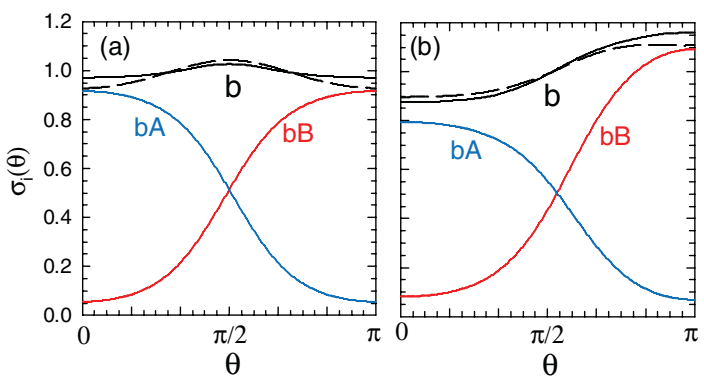

FIG. 5 (color). Grafting distributions, $\sigma_{i}(\theta)$, for a nanoparticle at (a) $z_{p} / D=0.0$ and (b) 0.1 . Solid curves are for the $A$ (blue), $B$ (red) and total (black) chains of a mixed brush, and the dashed curve is for a random-copolymer brush.

over which the particles prefer the interface is reduced to $0.2 \lesssim f_{b} \lesssim 0.8$. The reduction is not as great as suggested by the experiments, which found that a random-copolymer brush of 40\% PS was insufficient to remove particles from the P2VP domains. However, Kim et al. acknowledged that the grafting densities of their random-copolymer brushes were considerably less than those of their mixed brushes, and this may have resulted in a small amount of contact between the gold particles and the block copolymer, which is known to strongly favor the P2VP domains.

Although our calculation agrees with experiment, there are still further issues to consider. For instance, it is uncertain if the experimental samples were fully equilibrated; the measured particle distributions could be partially influenced by the sample history, in particular, the solvent annealing. Thus it would be interesting to theoretically investigate the effects of solvent. Furthermore, the polymer ligands forming the brush may be too short to treat as perfectly flexible Gaussian chains, and so we could consider modeling them with the wormlike chain model for semiflexible chains [17]. We could also include polymer interactions with the bare particle, as well as various other effects such as polydispersity, conformational asymmetry, compressibility, and so on. Another advantage of the theory is the ease with which it allows us to investigate modifications to the system, as it has the ability to treat brushes of almost any type imaginable. For nongold particles, the brushes may become immobile, but this can be readily treated by altering the expression for $q^{\dagger}(\mathbf{r}, \alpha)$ [18].
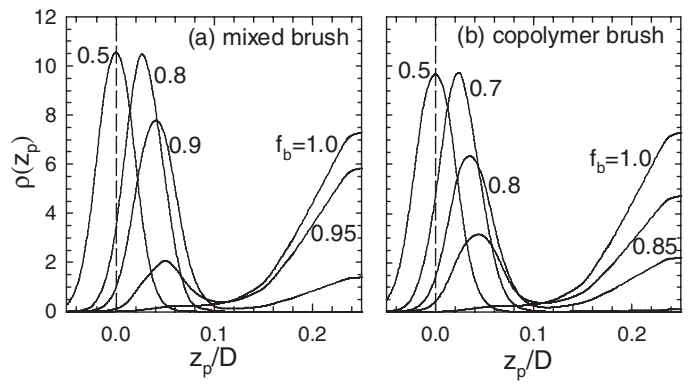

FIG. 6. Nanoparticle distribution, $\rho\left(z_{p}\right)$, for (a) mixed and (b) random-copolymer brushes of various compositions, $f_{b}$.
In summary, we have developed the first quantitative theoretical method capable of examining the effect of grafted brushes on the equilibrium distribution of spherical nanoparticles in a block-copolymer lamellar phase, by implementing self-consistent field theory (SCFT) with a new multi-coordinate-system (MCS) scheme. The method was applied to recent experiments by Kim et al. [12], where mixed and random-copolymer brushes were used to position gold nanoparticles at the interfaces of a PS-P2VP lamellar nanostructure. Consistent with the experiments, we predict that for equivalent conditions (i.e., polymerization $\alpha$, grafting density $V_{b}$, and composition $f_{b}$ ), the mixed brushes are significantly more effective than the random-copolymer brushes at positioning nanoparticles at the interface. As suggested by Kim et al., this happens because the unlike chains of the mixed brush, given sufficient time, segregate to opposite hemispheres creating Janus nanoparticles. Our calculation also has the scope to treat brushes or block copolymers of any type, to include extra components such as solvent, to account for polymer-particle interactions and so on. The MCS scheme itself is even more versatile, and could facilitate all sorts of new SCFT calculations.

We thank Ed Kramer and Bumjoon Kim for valuable discussions and EPSRC for financial support.

[1] A. C. Balazs, T. Emrick, and T. P. Russell, Science 314, 1107 (2006).

[2] M. R. Bockstaller and E. L. Thomas, Phys. Rev. Lett. 93, 166106 (2004); M. R. Bockstaller, R. Kolb, and E.L. Thomas, Adv. Mater. 13, 1783 (2001).

[3] H. K. Kang et al., Phys. Rev. Lett. 100, 148303 (2008).

[4] W. A. Lopes and H. M. Jaeger, Nature (London) 414, 735 (2001).

[5] S. C. Warren et al., Science 320, 1748 (2008).

[6] M. R. Bockstaller, A. Mickiewicz, and E. L. Thomas, Adv. Mater. 17, 1331 (2005).

[7] R. J. Spontak et al., Nano Lett. 6, 2115 (2006).

[8] M. R. Bockstaller, Y. Lapetnikov, S. Margel, and E.L. Thomas, J. Am. Chem. Soc. 125, 5276 (2003).

[9] J. U. Kim and B. O'Shaughnessy, Phys. Rev. Lett. 89, 238301 (2002).

[10] Y. Lin et al., Nature (London) 434, 55 (2005).

[11] J. J. Chiu et al., J. Am. Chem. Soc. 127, 5036 (2005).

[12] B. J. Kim et al., Langmuir 23, 12693 (2007).

[13] S. W. Sides, B. J. Kim, E. J. Kramer, and G. H. Fredrickson, Phys. Rev. Lett. 96, 250601 (2006).

[14] F. Teràn Arce, M.E. Vela, R.C. Salvarezza, and A.J. Arvia, Electrochim. Acta 44, 1053 (1998). S.-I. Imabayashi, D. Hobara, and T. Kakiuchi, Langmuir 17, 2560 (2001).

[15] R. B. Thompson, V. V. Ginzburg, M. W. Matsen, and A. C. Balazs, Science 292, 2469 (2001).

[16] M. W. Matsen and R. B. Thompson, Macromolecules 41, 1853 (2008).

[17] M. W. Matsen, J. Phys. Condens. Matter 14, R21 (2002).

[18] J. R. Roan, Phys. Rev. Lett. 87, 059902(E) (2001). 